\title{
The Development of Swirling Decaying Laminar Flow in an Annular Pipe
}

\author{
Baiman Chen $^{1 *}$, Frank G.F. Qin ${ }^{1}$, Youyuan Shao ${ }^{1}$, Hanmin Xiao ${ }^{1}$, Simin Huang ${ }^{1}$ and Kelvin $\mathrm{Ho}^{2}$ \\ ${ }^{1}$ Key Laboratory of Distributed Energy Systems of Guangdong Province, College of Chemical Engineering and Energy Technology, \\ Dongguan University of Technology, Dongguan 523808, China \\ ${ }^{2}$ The University of Nottingham, Faculty of Engineering, Jalan Broga, 43500, Semenyih, Selangor Darul Ehsan, Malaysia
}

\begin{abstract}
Work on the hydrodynamic entry length of pipe and duct flow has been well studied over the years. The assumption of fully developed flows is commonly used in many practical engineering applications (e.g. Moody's chart). For laminar axial pipe flow, the hydrodynamic entry length can be found through the monomial proposed by Kays, Shah and Bhatti (KSB) $(\mathrm{Lh}=0.056 \mathrm{ReDh})$. In contrast, several approximations exist for fully turbulent flows (i.e. $10 \mathrm{Dh}-150 \mathrm{Dh}$ ). Through theoretical and numerical investigations, the hydrodynamic entry length for swirling decaying pipe flow in the laminar regime is investigated. It was found that, the development length Lh for the axial velocity profile changes when a tangential component is added to the mean flow. The reduction in the hydrodynamic length was found to be dependent on the inlet swirl angle $\theta$. The results indicate that a modification can be made on the KSB equation for two-dimensional swirling annular pipe flow.
\end{abstract}

\section{Introduction}

The entry length of axial pipe flow has been well studied over the years [1-3]. The predictability of the entry length in pipes has benefited many practical engineering applications e.g. the estimation of wall friction factor in the fully developed region through the Moody Diagram [4], heat transfer for fully developed pipe flows [5] etc. The entry length for pipe flow is characterized by the boundary layer and the irrational core region. For the case of laminar axial flow, the velocity profile attains a fully developed state $(\partial \mathrm{u}(\mathrm{r}, \mathrm{x})) / \partial \mathrm{x}=0$, at $\mathrm{Lh}$, the hydrodynamic entry length. However useful the said equations may be, it has been limited to axial flow pipe flows. It would be an interesting attempt to adapt such equations to twodimensional flow, namely swirling/vortex pipe flow.

Swirling flow in pipes and ducts are found in many engineering applications [6, 7] (e.g. swirl tubes, vortex tubes, hydrocyclone, swirl combustors, pipe lines, mixers, flow guiders etc.). The work on various passive swirlers can be found in the wide literature [8-12].Kelvin et. al. [13-15] investigated the flow dynamics is swirling decaying flows in an annular pipe using both experimental and numerical techniques. The use of axial swirl vanes produced decaying swirl flows that can be defined using the swirl number $\mathrm{S}$ as functions of Re, pipe length and the vane angle. The focus of this work is to apply the numerical model developed in [7, 15] to investigate the effect of swirling flow towards the hydrodynamic entry length Lh inside and annular pipe.

\begin{tabular}{|c|c|c|c|}
\hline \multicolumn{4}{|c|}{ Nomenclature } \\
\hline A & Area, $\mathrm{m}^{2}$ & $\mathrm{u}, \mathrm{v}, \mathrm{w}$ & $\begin{array}{l}\text { Velocity, } \\
\mathrm{m} \cdot \mathrm{s}^{-1}\end{array}$ \\
\hline $\begin{array}{l}\text { A } \\
*\end{array}$ & $\begin{array}{l}\text { Aspect ratio, } \\
\mathrm{A}^{*}=\mathrm{z} / \mathrm{Dh}\end{array}$ & $\mathrm{V}$ & $\begin{array}{l}\text { Volume, } \\
\mathrm{m}^{3}\end{array}$ \\
\hline $\mathrm{D}$ & $\begin{array}{l}\text { Diameter, } \\
\mathrm{mm}\end{array}$ & $\alpha$ & $\begin{array}{l}\text { Thermal } \\
\text { diffusivity } \\
\text { of solid, } \\
\mathrm{m}^{2} \mathrm{~s}^{-1} \\
\text { Turbulenc }\end{array}$ \\
\hline $\mathrm{e}$ & $\begin{array}{l}\text { Diametric } \\
\text { clearance( } \mathrm{Dh} / \\
\text { 2), } \mathrm{mm}\end{array}$ & $\varepsilon$ & $\begin{array}{l}\mathrm{e} \\
\text { dissipatio } \\
\mathrm{n} \text { rate, } \\
\mathrm{m}^{2} \mathrm{~s}^{-3}\end{array}$ \\
\hline $\mathrm{L}$ & Length, mm & $\mu$ & $\begin{array}{l}\text { Dynamic } \\
\text { viscosity, } \\
\mathrm{kg} \mathrm{m}^{-1} \mathrm{~s}^{-1}\end{array}$ \\
\hline $\mathrm{P}$ & Pressure, $\mathrm{Pa}$ & $\rho$ & $\begin{array}{l}\text { Density, } \\
\mathrm{kgm}^{-3}\end{array}$ \\
\hline $\begin{array}{c}\mathrm{R}, \\
\mathrm{r}\end{array}$ & Radius, mm & $\theta$ & $\begin{array}{l}\text { Vane } \\
\text { angle }\end{array}$ \\
\hline $\mathrm{S}$ & $\begin{array}{l}\text { Swirl number, } \\
\mathrm{S}=\mathrm{v} / \mathrm{W}\end{array}$ & $\omega$ & $\begin{array}{l}\text { Rotational } \\
\text { speed, } \mathrm{s}^{-1}\end{array}$ \\
\hline $\mathrm{t}$ & Time, $\mathrm{s}$ & & \\
\hline
\end{tabular}

* Corresponding author: chenbm@dgut.edu.cn 


\section{Methods}

The experimental setup in Kelvin et. al. [15] for decaying flow is modelled using the commercial codes from ANSYS CFX 12.0. The flow field for different vanes was investigated by Baiman et. al. [14]. The focus of this work is to apply the same numerical model to investigate the effect of swirling flow towards the hydrodynamic entry length, Lh. In-depth information on the simulation model can be found in [7,13-15]. The equations (eq. 1-2) were solved for three dimensional laminar flows ( $\mu$ eff $=$ $\mu ; \mu \mathrm{t}=0)$.

$$
\nabla \cdot\left(\rho_{f} U\right)=0
$$

The simplified time-independent non-viscous NavierStokes equation:

$$
(\nabla \cdot U) \rho_{f} U-\mu_{e f f} \nabla^{2} U+\nabla P-\nabla \cdot\left(\mu_{e f f} \nabla U\right)^{T}=0
$$

Where

$$
\mu_{\text {eff }}=\mu+\mu_{t}
$$

The fluid domain of the annular pipe and inlet swirler was modelled (figure 1) and discretised using semi structured mesh [7].Water ( $\rho=1000 \mathrm{~kg} / \mathrm{m} 3 ; \mu=0.001 \mathrm{~Pa} \mathrm{~s}$ ) was used as the working fluid of the system. The annular tube has a length of $1000 \mathrm{~mm}(\mathrm{Di}=30 \mathrm{~mm}$; $\mathrm{Do}=46 \mathrm{~mm}$; $\mathrm{Dh}=16 \mathrm{~mm})$. Each of the vane $\left(30^{\circ}, 40^{\circ}\right.$ and $\left.60^{\circ}\right)$ was investigated using an average of $1 \times 106$ nodes.

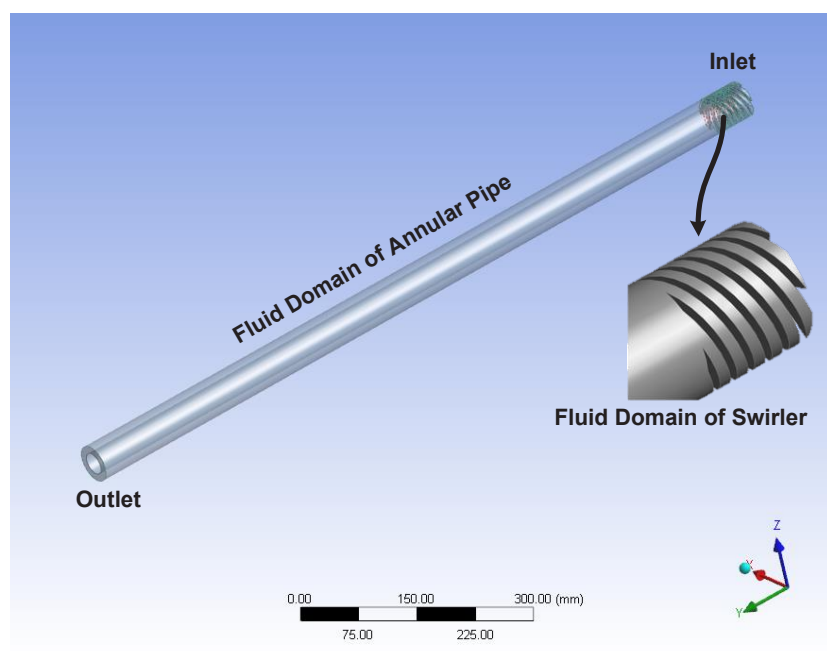

Figure 1. Computational Domain.

\section{Numerical Results}

The onset of this work was to investigate the effect of two-dimensional flow towards the hydrodynamic entry length. The numerical predictions for all three vane angles $\left(30^{\circ}, 45^{\circ}\right.$ and $\left.60^{\circ}\right)$ are shown in figure 2(a)-(f) along the annular pipe $\left(A^{*}=1-52\right)$ and for two different Reynolds number ( $\mathrm{Re}=347$ \& 694). The bias of the velocity profile towards the outer pipe is caused by the swirling momentum and reduces as the vane angle increases. The symmetry of the velocity profile increase as the Swirl number decreases [14] and attains a fully developed state when $A^{*}$ reaches a certain length. The value of $A^{*}$ can then be used to estimate the hydrodynamic entry length, Lh.

From the work of Kays [1] and Shah and Bhatti [2] (KSB), it is known that the hydrodynamic entry length (Lh) can be found using the following equation.

$$
\frac{L_{h}}{D_{h}}=0.056 \mathrm{Re} \quad \text {; For laminar internal pipe flow }
$$

$$
L_{h}=f\left(\frac{D_{h}}{2}, \mu, \rho, U, V, \theta\right)
$$

Using the Buckingham $\pi$-Theorem eq. 4 can be refined to eq. 5

$$
2 \frac{L_{h}}{D_{h}}=f\left(\frac{\rho U D_{h}}{\mu}, \operatorname{Sin} \theta\right)
$$

The hydrodynamic entry length can be normalized using the hydrodynamic diameter of the annulus $(\mathrm{A} *=\mathrm{Lh} / \mathrm{Dh})$.

The Reynolds number is calculated using axial velocity U.For swirling decaying flow the ratio between $\mathrm{U}$ and $\mathrm{V}$ can be related through the angle $\theta$. [14].Dh/2 is used for an annular pipe because a developed velocity profile will only occupy $\mathrm{Dh} / 2$ in contrast to $\mathrm{Dh}$ for pipe.

$$
\frac{L_{h}}{D_{h}}=\frac{A}{2} \cdot \operatorname{Re}^{\alpha} \cdot \operatorname{Sin} \theta^{\beta}
$$

Assuming that $\alpha \approx 1[1,2]$, the Reynolds number will have the same power as eq. 3. From the work of Baiman et. al. [7], the Resultant Reynolds number can be defined as $\operatorname{Re} / \sin \theta(\beta \approx-1)$.

$$
\frac{L_{h}}{D_{h}}=0.028 \frac{\mathrm{Re}}{\operatorname{Sin} \theta}
$$

Equation 6 shows that, the hydrodynamic entry length for swirling decaying flow in an annular duct can be obtained through a modified version of the monomial proposed by Kays, Shah and Bhatti $[1,2]$. A comparison between the Lh predicted using the numerical model and the correlation in eq. 6 is shown in figure 3. The comparison indicates that the hydrodynamic entry length of a two-dimensional swirling decaying flow in an annular pipe is a function of the Reynolds number and the vane angle, $\theta$.

\section{Conclusion}

The validated numerical model from a previous work [13] was used to study the effect of two-dimensional swirling flow towards the hydrodynamic entry length in an annular pipe. The monomial that was devised using the $\pi$ Theorem managed to predict the Lh and was comparable to the numerical predictions. However, the universality of the modified monomial and its dependency to other governing parameters (e.g. Swirl number, geometrical configurations, pressure drops etc) still requires additional work and is the subject of future work. 


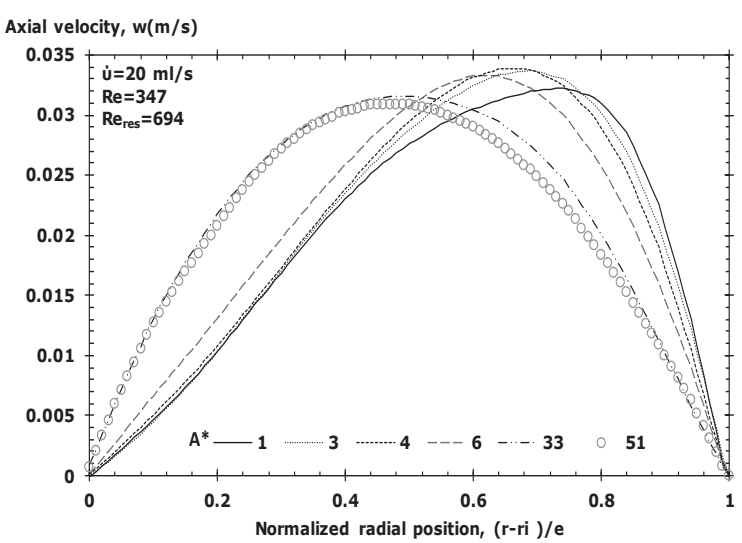

(a)

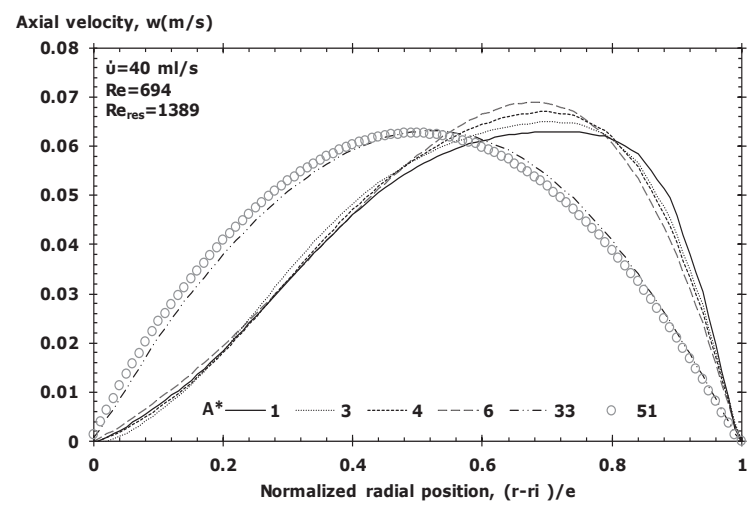

(b)

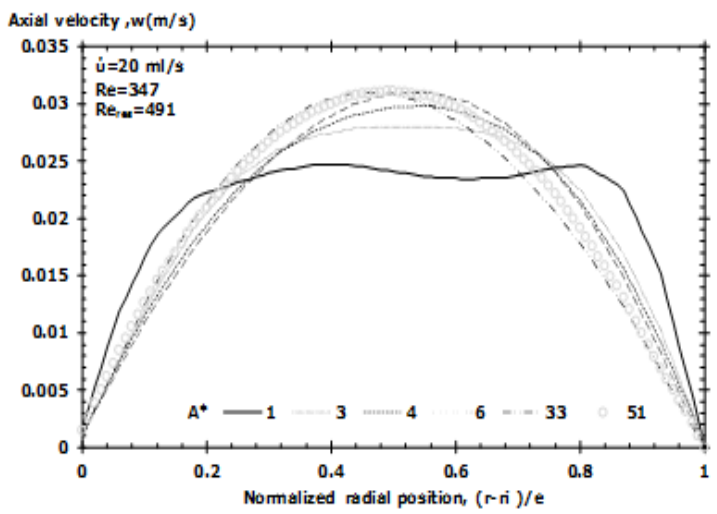

(c)

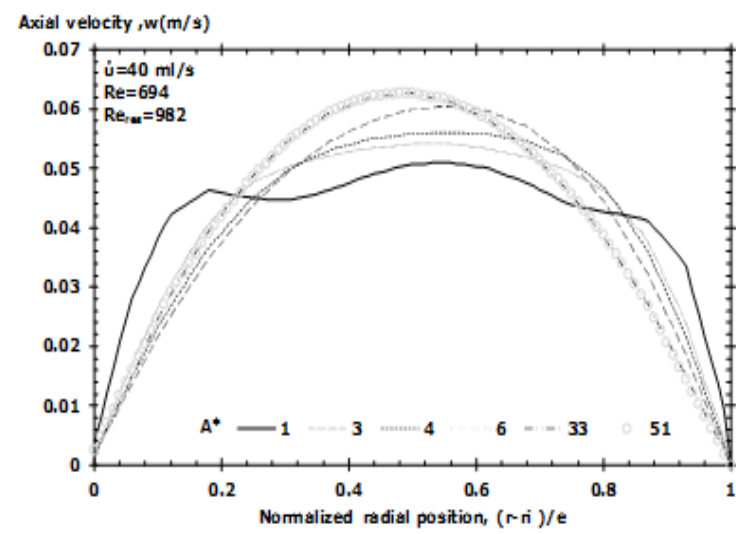

(d)

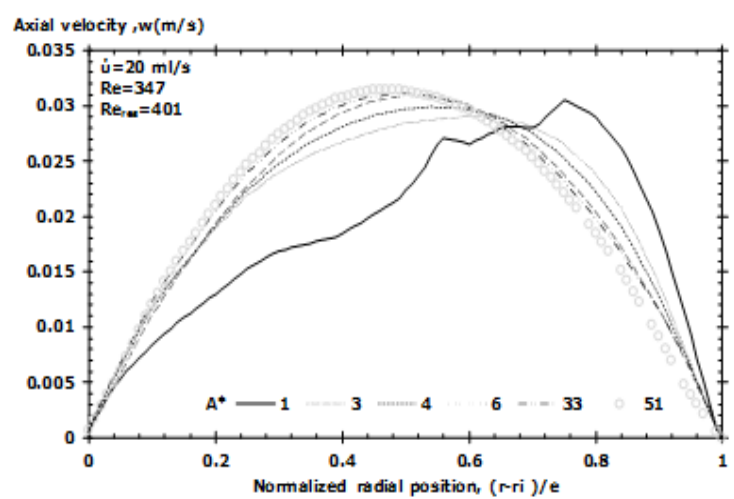

(e)

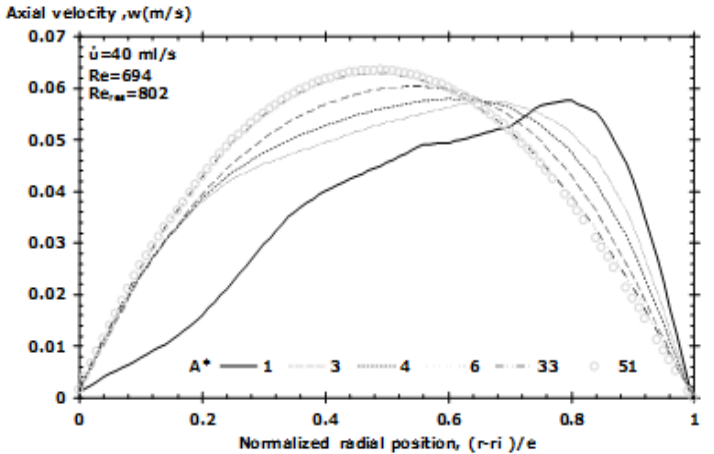

(f)

Figure 2. The development of the axial velocity profile along the annular pipe for (a-b) $30^{\circ}$ vanes (c-d) $45^{\circ}$ vanes (e-f) $60^{\circ}$ vanes.

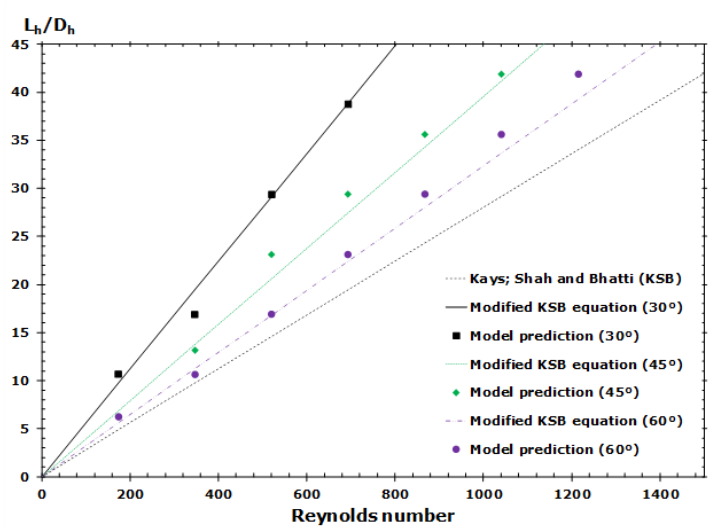

Figure 3. The hydrodynamic entry length for axial and swirling decaying pipe flow.

\section{Acknowledgments}

This work was supported by the China National Natural Science Foundation Project (No. 51406036) and Guangdong Special Innovation Project of University (No. 2016KTSCX138).

\section{References}

1. W.M. Kays, Numerical solutions for laminar-flow heat transfer in circular tubes, Trans. ASME. 77 (1955) 1265-1274. 
2. R.K. Shah, M.S. Bhatti, "Laminar convective heat transfer in ducts," in Handbook of single-phase conective heat transfer, John-Wiley, 1987.

3. K. Lien, J.P. Monty, M.S. Chong, A. Ooi, The Entrance Length for Fully Developed Turbulent Channel Flow, in:15th Australas. Fluid Mech. Conf., 2004: pp.

$1-4$. http://sydney.edu.au/engineering/aeromech/15afmc/p roceedings/papers/AFMC00093.pdf.

4. L.F. Moody, Friction factors for pipe flow, Trans $\begin{array}{llll}\text { ASME. } & 66 & (1944) & 671-684 .\end{array}$ doi:10.1017/S0022112004009796.

5. A. Bejan, Convection heat transfer, 3rd ed., Wiley, New Jersey, 2004.

6. R.J. Goldstein, W.E. Ibele, S. V Patankar, T.W. Simon, T.H. Kuehn, P.J. Strykowski, et al., Heat transfer--A review of 2005 literature, Int. J. Heat Mass Transf. 53 (2010) 4397-4447. http://www.sciencedirect.com/science/article/B6V3H -50GJ9DT-

2/2/2826de31da348507c65d8dea83846308.

7. B. Chen, K. Ho, Y.A. Abakr, A. Chan, Fluid dynamics and heat transfer investigations of swirling decaying flow in an annular pipe Part 1: Review, problem description, verification and validation, Int. J. Heat Mass Transf.

(2015). doi:10.1016/j.ijheatmasstransfer.2015.07.129.

8. R. Hreiz, C. Gentric, N. Midoux, Numerical investigation of swirling flow in cylindrical cyclones, Chem. Eng. Res. Des. 89 (2011) 2521-2539. doi:10.1016/j.cherd.2011.05.001.

9. T. Bali, B.A. Sarac, B. Ayhan, Experimental investigation of decaying swirl fl ow through a circular pipe for binary combination of vortex generators $\hat{i}$, Int. Commun. Heat Mass Transf. 53 (2014)

$174-179$. doi:10.1016/j.icheatmasstransfer.2014.02.030.
10. M. Ahmadvand, A.F. Najafi, S. Shahidinejad, An experimental study and CFD analysis towards heat transfer and fluid flow characteristics of decaying swirl pipe flow generated by axial vanes, Meccanica. 45 (2010) 111-129. doi:10.1007/s11012-009-9228-9.

11. C. Biegger, C. Sotgiu, B. Weigand, Numerical investigation of flow and heat transfer in a swirl tube, Int. J. Therm. Sci. 96 (2015) 319-330. doi:10.1016/j.ijthermalsci.2014.12.001.

12. C. Biegger, B. Weigand, Detached Eddy Simulation of Flow and Heat Transfer in Swirl Tubes, in: High Perform. Comput. Sci. Eng. '15, Springer International Publishing, Cham, 2016: pp. 449-461. doi:10.1007/978-3-319-24633-8_29.

13. B. Chen, K. Ho, F.G.F. Qin, R. Jiang, Y.A. Abakr, A. Chan, Validation and Visualization of Decaying Vortex Flow in an Annulus, 7th Int. Conf. Appl. Energy - ICAE2015. 75 (2015) 3098-3104. doi:10.1016/j.egypro.2015.07.640.

14. B. Chen, K. Ho, Y.A. Abakr, A. Chan, Fluid dynamics and heat transfer investigations of swirling decaying flow in an annular pipe Part 2: Fluid flow, Int. J. Heat Mass Transf. (2016). doi:10.1016/j.ijheatmasstransfer.2016.01.069.

15. K. Ho, Y.A. Abakr, A. Chan, An experimental set-up for investigating swirling decaying flow in an annular pipe, Int. Commun. Heat Mass Transf. 38 (2011) 1253-1261. 